\title{
The Standard Refuelling Facility Expansion Problem: A Case Study in Australia
}

\author{
$\underline{\text { Juan Calle }}^{\mathrm{a}}$, Roberto Perez-Franco ${ }^{\mathrm{a}}$, Vicky Mak-Hau ${ }^{\mathrm{b}}$, Danilo Abril ${ }^{\mathrm{c}}$ and Daniel Rojas $^{\mathrm{d}}$ \\ ${ }^{a}$ Centre for Supply Chain and Logistics, Deakin University, Burwood, Vic 3125, Australia \\ ${ }^{\mathrm{b}}$ School of Information Technology, Deakin University, Burwood, Vic 3125, Australia \\ ${ }^{\mathrm{c}}$ Independent Researcher, Colombia \\ ${ }^{\mathrm{d}}$ EIA University, Colombia \\ Email: jcallesalazar@deakin.edu.au
}

\begin{abstract}
Refuelling facilities are critical infrastructure for road freight transport networks, since every vehicle must refuel in order to move freight. Recent advances in alternative fuel (alt-fuel) vehicles have made the location of refuelling facilities even more critical, since the driving range of alt-fuel vehicles is less than for traditional fuel-powered vehicles (Deb et al., 2018). Transport companies have decided to establish their own refuelling facilities in order to guarantee the feasibility of their vehicles' movements, as well as to optimise the refuelling process. This article presents a methodology to assist transport companies to locate their own refuelling facilities. It first proposes a methodology to convert GPS data into trips to understand where the vehicles are moving, then uses this information as an input for a customised Mixed Integer Programming (MIP) model that suggests potential locations for refuelling facilities. The methodology is applied to a case study in Australia (the Melbourne-Sydney corridor).
\end{abstract}

The transport company that is the subject of this case study provided all the required data to run the model ${ }^{1}$. Their motivation to do the analysis in this corridor was the high amount of fuel refuelled at retailers (refuelling facilities of third parties), which is in the order of tens of millions of litres of fuel per year. They decided to investigate the movements of the fleet to evaluate the business viability to locate their own-refuelling facilities in the corridor. The main advantage for the transport company to have their own-refuelling facilities was to enable the purchase of fuel at wholesale prices directly from fuel suppliers, thus improving the efficiency of refuelling operations, since strategically located refuelling facilities could mean that the vehicles spend less time in the refuelling operation.

For this particular case study, the model suggests that only by improving the refuelling decision taken by the fleet (where and how much to refuel) $2.3 \%$ of savings (representing hundreds of thousands of dollars) could be obtained out of the total refuelling cost of the company in the analysed corridor. After doing a sensitivity analysis on the fuel consumed in the corridor, it is suggested that only one own-refuelling facility should be located, since the location of two refuelling facilities would not be an optimal solution if the fuel consumed in the corridor is reduced by more than $20 \%$.

The MIP presented in this article is a contribution to the flow refuelling location 1 iterature, $s$ ince it adds differential prices of the fuel in each refuelling facility as a driver to locate refuelling facilities. Another contribution is the novel procedure presented in this paper to process and convert large amounts of GPS data into trips.

${ }^{1}$ All data referring to the company has been masked to protect its confidentiality

Keywords: Transport, flow refuelling location, GPS data transformation, mixed integer programming 


\section{INTRODUCTION}

Refuelling facilities are critical infrastructure for road freight transport networks, since every vehicle must refuel in order to move freight. Recent advances in alt-fuel vehicles have made the location of refuelling facilities even more critical, since the driving range of alt-fuel vehicles is less than for traditional fuel-powered vehicles (Deb et al., 2018). Transport companies have decided to establish their own refuelling facilities in order to guarantee the feasibility of their vehicles' movements, as well as to optimise the refuelling process. This article presents a methodology to assist transport companies to locate their own refuelling facilities. It first proposes a methodology to convert GPS data into trips to understand where the vehicles are moving, then uses this information as an input for a customised Mixed Integer Programming (MIP) model that suggests potential locations for refuelling facilities. The methodology is applied to a case study in Australia.

The problem of locating refuelling facilities falls in the category of facility location problems, which has been extensively studied in the Operations Research literature. This problem was first reviewed by Kuby and Lim (2005) considering two important factors into the location decisions: the moving demand (vehicles), and the necessity to guarantee that the vehicles could finish their trips without running out of fuel. After this seminal work, several papers have developed modelling improvements and extensions of the flow refuelling location problem (Deb et al., 2018). However, to the extent of our knowledge the research in the flow refuelling location problem has not considered the fuel price at the station (refuelling facility) as a relevant parameter in the location decisions, which for the transport company subject of the case study is an important driver to locate a new station.

The underlying framework of the MIP presented in this work, comes from the vehicle refuelling literature. The vehicle refuelling problem deals with the decisions of where and how much a vehicle must refuel in each station along the shortest path between a determined origin and destination to minimise the total refuelling cost (Suzuki, 2008). Combining this framework with the elements from the flow refuelling location literature allowed us to model the Standard Refuelling Facility Expansion Problem (SRFEP).

The rest of the document is organised as follows: section 2 presents the customised algorithm developed to convert the GPS data into trips with defined origin and destination. Section 3 presents the elements of what we called the Standard Refuelling Facility Expansion Problem (SRFEP) and the MIP associated to it. Section 4 presents the numerical analysis of the case study. Section 5 presents the conclusions of the case study. Finally, section 6 presents areas of future research related to the SRFEP.

\section{Processing the GPS DATA}

At the time of the case study, the company did not have a consolidated transactional system of their whole operation. Therefore, the best approach to understand how the vehicles that refuelled in the corridor were moving was to study the GPS data generated by the vehicles, which reported the position of the vehicle at specific moments (time stamp). Algorithms to process GPS data have been widely used for passenger transport analysis and recently they have also been used to understand freight movements by transport planning agencies (Thakur et al., 2015). However, to the extent of our knowledge GPS data has never previously been used to understand the origin and destination of a trip when a vehicle refuels in a given corridor.

The company provided three different data-sets: vehicles GPS data, refuelling transactions data, and station data. Each register of the vehicles' GPS data is named as a telemetry point (TP) which contains a Vehicle ID, Time Stamp (TS) at the level of seconds, and coordinates. Each register of the refuelling transactions data contains the vehicle ID, station ID, refuelling date at the level of days and refuelling quantity in litres. Finally, the service station data-set contains information about the station ID and coordinates.

The algorithm to convert the GPS data into trips is as follows:

1. Find the closest telemetry point to refuelling transaction (CTP).

1.1. Calculate the time difference between the TS of the TP and the date of the refuelling transaction.

1.2. For those TP that occurred the same day as the refuelling transaction calculate the distance to the station in which occurred the refuelling transaction.

1.3. The TP with smallest distance to the refuelling transaction is chosen as the CTP.

2. Find origin and destination associated to each refuelling transaction.

2.1. Sequence TP in chronological order for each vehicle 
J. Calle, et al, The standard refuelling facility expansion problem: A case study in Australia

2.2. Classify each TP if it is inside or outside the corridor.

2.3. The previous TP before the CTP which previous TP is outside the corridor is defined as the origin of the trip associated to the refuelling transaction

2.4. The previous TP after the CTP wich following TP is outside the corridor is defined as the destination of the trip.

3. The trips associated to refuelling transactions, in which the origin and destination of the trip is the same point are considered trips with U-turn. For those trips choose the leg of the trip in which the vehicle refuelled to redefine origin or destination.

The details of the algorithm that identifies U-turn and recalculate the origin and destination for trips with U-Turn are presented as supplementary material (Calle et al., 2021).

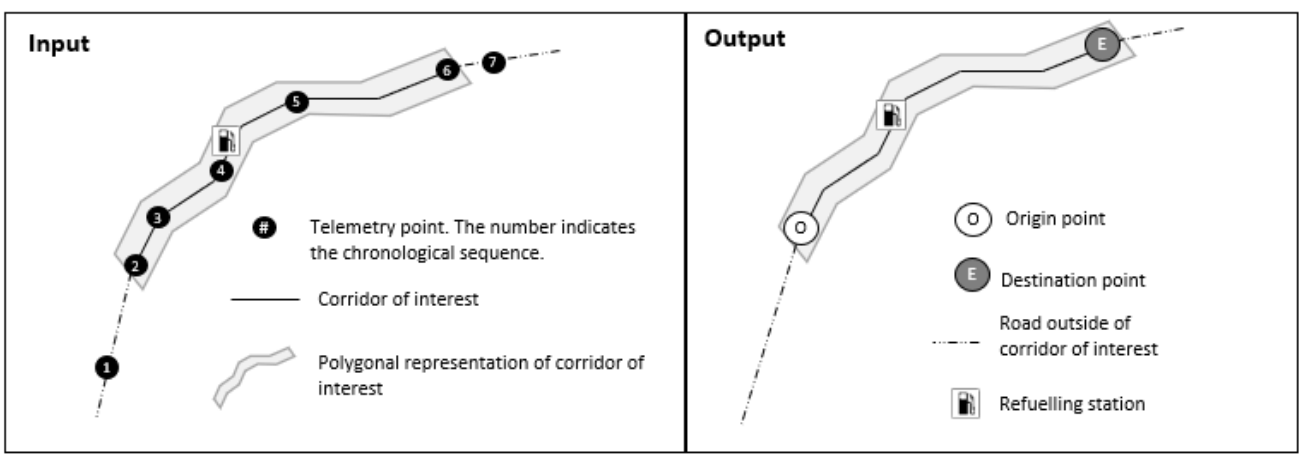

Figure 1. Graphical representation of the GPS data process

After processing the GPS data, it was possible to estimate the origin and destination of the trips that refuelled $70 \%$ of the fuel consumed in the corridor in the analysed planning horizon. This lack of data was because not all the vehicles had a GPS system at the moment of the study and other vehicles did not report a CTP close enough to a refuelling station to be considered as a hint to estimate the origin and destination of the trip. To estimate the origin and destination of the rest of the trips that consumed fuel in the corridor, it was assumed that this group of vehicles moved proportionally in the same paths as the vehicles that had enough information to define the origin and destination of the trip.

\section{Mixed Integer Programming model (MIP)}

We defined our problem as the Standard Refuelling Facility Expansion Problem (SRFEP). The purpose of the MIP model for the SRFEP is to evaluate the strategical decisions of where to locate new refuelling stations. To achieve this purpose the Flow Refuelling Location (FRL) literature has not only taken into account the variables to model the strategical decisions, but also the operational decisions that define the refuelling plan for each type of vehicle. Some authors have considered this refuelling plan explicitly (Wang \& Lin, 2009), while others have done it implicitly (MirHassani \& Ebrazi, 2013). The operational detail in the FRL literature (where and how much to refuel) is important because it is necessary to guarantee in the location decisions that the vehicles have enough refuelling option in their trips to not run out of fuel. However, in the case of the SRFEP the feasibility of the trip is not a concern because there are enough retail stations to guarantee the feasibility of the trip of each type of vehicle, but for the SRFEP is important that the model guarantee that the potential stations to locate can deliver the amount of fuel necessary to justify the investment. It is achieved through capturing the details of the refuelling plan of each type of vehicle having into account the fuel-tank capacity and the fuel inventory before refuelling.

The SRFEP can be stated as follows:

Given a set of paths $\mathcal{P}$, which are travelled by a number of vehicles of a given type $v$. It must be defined the refuelling plan for each type of vehicle $v$ in each path $p$, it means the amount refuelled at each station $i$ by vehicles of type $v$ travelling through path $p$ to guarantee that the type of vehicle does not run of fuel in its travel. As well as, if a potential station must be built or not, and which should be its extra units of capacity $\left(u_{i}\right)$ to minimize the total refuelling and location costs. It must also be taken into account that potential stations 
J. Calle, et al, The standard refuelling facility expansion problem: A case study in Australia

has a limited capacity to be determined and existing stations are assumed to have infinite capacity, since they are owned by third parties.

Each path is defined by a set of nodes $\left\{O_{p}, s_{1}, s_{2}, \ldots, s_{n_{p}}, E_{p}\right\}$, where $O_{p}$ is the origin of the path $p, s_{i}$ is a station along the path and $E_{p}$ is the destination of the path $p$. Each station has associated two points, a diversion point and an actual point. The diversion point is a virtual point to calculate the deviation distance from the main path to go to refuel to the station, while the actual point is the geographical point in which the refuelling operation takes place (see Fig. 2).

An additional assumption of the SRFEP is that the total refuelling quantity of each type of vehicle $v$ in path $p$ is known in advance. This is done to be able to use the model as a tool to compare what actually happened versus what would happen if new stations were added into the corridor. Table 1 shows the description of the notation used to represent the model for the SRFEP. The MIP to solve the SRFEP is given by:

Table 1. Notation of the MIP for the RFEP

\begin{tabular}{|c|c|c|}
\hline \multicolumn{3}{|r|}{ Sets } \\
\hline Notation & Index & Description \\
\hline $\mathcal{P}$ & $p$ & Paths \\
\hline $\mathcal{P}_{i}$ & $p$ & Paths associated to station $i$ \\
\hline $\mathcal{S}$ & $i$ & All stations \\
\hline $\mathcal{S}^{P O T}$ & $i$ & Potential stations \\
\hline $\mathcal{S}_{p}$ & $i$ & Stations along path $p$ \\
\hline $\mathcal{V}$ & $v$ & Types of vehicles \\
\hline $\mathcal{V}_{p}$ & $v$ & $\begin{array}{l}\text { Types of vehicles that travel } \\
\text { along path } p\end{array}$ \\
\hline \multicolumn{3}{|r|}{ Variables } \\
\hline Notation & Domain & Description \\
\hline$q_{i, v, p}$ & $\mathbb{R}^{+}$ & $\begin{array}{l}\text { Amount to refuel for type of vehicle } v \text { travelling } \\
\text { through path } p \text { at station } i\end{array}$ \\
\hline$r_{i, v, p}$ & $\mathbb{R}^{+}$ & $\begin{array}{l}r_{i, v, p}=1 \text { indicates that type of vehicle } v \text { travelling } \\
\text { through path } p \text { refuels at station } i, 0 \text { otherwise }\end{array}$ \\
\hline$a_{i, v, p}$ & $\mathbb{R}^{+}$ & $\begin{array}{l}\text { Amount of fuel in the tank in type of vehicle } v \text { travelling } \\
\text { through path } v \text { when it arrives at station } i\end{array}$ \\
\hline$y_{i}$ & $\{0,1\}$ & $y_{i}=1$ indicates station $i$ is built, 0 otherwise \\
\hline$u_{i}$ & $\mathbb{Z}^{+}$ & Quantity of units of capacities to add to station $i$ \\
\hline
\end{tabular}

\begin{tabular}{|l|l|}
\hline \multicolumn{2}{|c|}{ Parameters } \\
\hline Notation & Description \\
\hline$Q_{v, p}^{v e h}$ & Number of vehicles of type $v$ travelling through path $p$ \\
\hline$K_{i}$ & $\begin{array}{l}\text { Capacity of station } i \text { in terms of the amount of fuel } \\
\text { delivered at the station }\end{array}$ \\
\hline$K_{i}^{u}$ & $\begin{array}{l}\text { Additional capacity the station } i \text { could have if it is } \\
\text { decided to add } 1 \text { additional unit of capacity }\end{array}$ \\
\hline$C_{i}^{\text {loc }}$ & Cost of locating station $i$ \\
\hline$C_{i}^{u c a}$ & Cost of one additional unit of capacity in station $i$ \\
\hline$P_{i}^{p r i}$ & Price of one unit of volume of fuel at station $i$ \\
\hline$C_{i, v, p}^{v a r}$ & $\begin{array}{l}\text { Variable cost different from the fuel of travelling } \\
\text { one unit of distance with vehicle type } v\end{array}$ \\
\hline$C_{v}^{o p p}$ & Opportunity cost of spending time refuelling vehicle $v$ \\
\hline$D_{i, p}^{o o p}$ & Distance out of the path $p$ of station $i$ \\
\hline$F_{v}^{\text {max }}$ & Capacity of the tank of type of vehicle $v$ \\
\hline$F_{v, p}^{r e f}$ & Capacity of the tank of type of vehicle $v$ \\
\hline$R_{v}^{\text {min }}$ & Minimum amount of fuel to refuel for type of vehicle $v$ \\
\hline$F_{i, j, v, p}^{c o n}$ & $\begin{array}{l}\text { Fuel consumption of type of vehicle } v \text { travelling } \\
\text { through path } p \text { between nodes } i \text { and } j\end{array}$ \\
\hline$F_{i, v, p}^{c o d}$ & $\begin{array}{l}\text { Fuel consumption of type of vehicle } v \text { travelling } \\
\text { through path } p \text { between station } i \text { and its diversion point }\end{array}$ \\
\hline
\end{tabular}

$$
\begin{aligned}
& \min \sum_{p \in \mathcal{P}} \sum_{i \in \mathcal{S}_{p}} \sum_{v \in \mathcal{V}_{p}} Q_{v, p}^{v e h}\left(P_{i}^{p r i} q_{i, v, p}+C_{v}^{o p p} r_{i, v, p}+D_{i, p}^{o o p} C_{i, v, p}^{v a r} r_{i, v, p}\right)+\sum_{i \in \mathcal{S}^{P O T}}\left(C_{i}^{l o c} y_{i}+C_{i}^{u c a} u_{i}\right) \\
& q_{i, v, p} \geq R_{v}^{\min } r_{i, v, p} \quad \forall p \in \mathcal{P}, v \in \mathcal{V}_{p}, i \in \mathcal{S}_{p} \\
& q_{i, v, p} \leq F_{v}^{\max } r_{i, v, p} \quad \forall p \in \mathcal{P}, v \in \mathcal{V}_{p}, i \in \mathcal{S}_{p} \\
& a_{i, v, p}+q_{i, v, p} \leq F_{v}^{\max } \quad \forall p \in \mathcal{P}, v \in \mathcal{V}_{p}, i \in \mathcal{S}_{p} \\
& a_{i+1, v, p}=a_{i, v, p}+q_{i, v, p}-\left(F_{i, i+1, v, p}^{c o n}+F_{i+1, v, p}^{c o d} r_{i+1, v}+F_{i, v, p}^{c o d} r_{i, v}\right) \quad \forall p \in \mathcal{P}, v \in \mathcal{V}_{p}, i \in \mathcal{S}_{p} \\
& \sum_{p \in \mathcal{P}_{i}} \sum_{v \in \mathcal{V}_{p}} Q_{v, p}^{v e h} q_{i, v, p} \leq K_{i}+K_{i}^{u} u_{i} \quad \forall i \in S^{P O T} \\
& r_{i, v, p} \leq y_{i} \quad \forall p \in \mathcal{P}, v \in \mathcal{V}_{p}, i \in S^{P O T} \\
& \sum_{i \in \mathcal{S}_{p}} q_{i, v, p}=F_{v, p}^{r e f} \quad \forall p \in \mathcal{P}, v \in \mathcal{V}_{p}
\end{aligned}
$$

The objective function (eq. 1) minimises the refuelling cost (first summation) plus location and expansion costs (second summation). Eq. 2 defines that each time a type of vehicle in a path refuels it must refuel at least $R_{v}^{\min }$. Eq. 3 defines that if a type of vehicle in a path goes to refuel to a station, the refuelling quantity can be at most the tank capacity of the vehicle, if the type of vehicle does not go to refuel, the refuelling quantity in the station must be zero. Eq. 4 defines the maximum refuelling quantity depending on the inventory in the 
fuel tank before refuelling. Eq. 5 refers to the inventory balance in each point of the path, if a type of vehicles in a path refuels in the station the balance is revised in the actual point, if the type of vehicle does not go to the station, the inventory balance is revised in the diversion point. Eq. 6 defines the capacity of a potential station to deliver fuel. If the standard capacity is not enough, extra units of capacity can be added to match the required capacity, one extra unit of capacity provides $K_{i}^{u}$ litres of additional capacity. Eq. 7 turns on the location variable if there is a refuelling operation in the potential station. Eq. 8 defines that the total refuelling quantity of a type of vehicle in a given path is already defined.

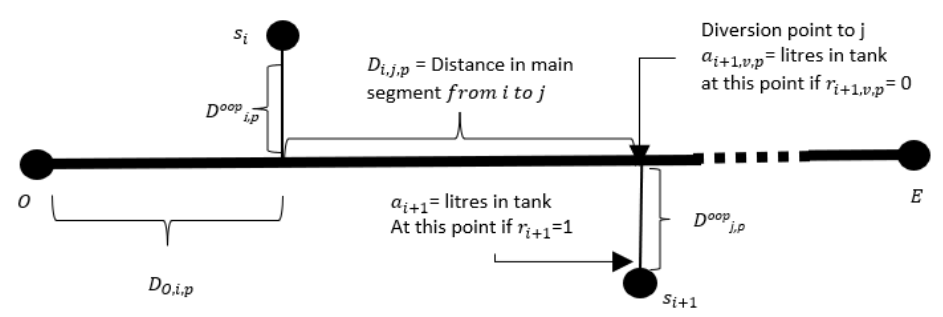

Figure 2. Representation of a path. Adapted from Suzuki (2008)

\section{NUMERICAL ANALYSIS}

\subsection{Input Summary}

The analysed corridor and some important reference points can be seen as supplementary material (Calle et al., 2021). It comprehends $732 \mathrm{~km}$ of the Hume Highway which is the main freight corridor between Melbourne and Sydney. The corridor expands from Craigieburn - Victoria, to Wilton - New South Wales.

The SRFEP is run with historical data considering a planning horizon of 1 year, which means that the quantity of vehicles of a given type that travel through a path $\left(Q_{v, p}^{v e h}\right)$ accounts for trips done in one year. To make the location and expansion cost equivalent for a year, we calculated for each of these values a cash flow for 10 years having into account the initial investment, the fixed annual operating cost and the salvage value at the end of the 10 years. This cash flow is discounted to the present with the WACC of the company and an equivalent annuity is calculated. The formulas used in this process are presented as supplementary material (Calle et al., 2021).

In the corridor Melbourne-Sydney, 21 million litres of fuel are refuelled in retailers per year. Locating a station with a capacity of 9.4 million litres/year will cost $\$ 108,859\left(K_{i}\right)$ and expanding its capacity by 2.3 million litres per year $\left(K_{i}^{u}\right)$ would cost $\$ 21,792$. The fuel price in the corridor is between 130c and 160c per litre. The potential station to locate would provide between $3 \mathrm{c}$ and $11 \mathrm{c}$ per litre of savings in respect to the surrounding retail stations in the corridor.

\subsection{Scenario Definition}

Baseline: this scenario tries to mimic the behaviour of the real system as closely as possible (Watson et al., 2013). To do this, the baseline does not include in the set of stations the potential stations to be located and some constraints are added to the model to force the model to behave as similar as possible to the real system. In the case of the SRFEP we added the following constraints to the original model to build the baseline:

$F_{i}^{l b} \leq \sum_{p \in \mathcal{P}_{i}} \sum_{v \in \mathcal{V}_{p}} Q_{v, p}^{v e h} q_{i, v, p} \leq F_{i}^{u b} \quad \forall i \in \mathcal{S}$

$F_{i}^{l b}$ and $F_{i}^{u b}$ represents $\pm 5 \%$ of the actual refuelling quantity in each station. We ran the model with this constraint and the error in the total cost vs real life cost was $0.08 \%$. The error was calculated as: $\frac{\left|z^{\text {real }}-z^{\text {model }}\right|}{z^{\text {real }}}$ $z^{\text {real }}$ is the objective function with real data, $z^{\text {model }}$ is the objective function of the baseline.

The baseline is also used to create a reference of comparison with the other scenarios.

Optimised baseline: this scenario removes from the baseline eq. 9 . This scenario is run to evaluate how much could the company save just by improving their refuelling decisions. This scenario does not include potential stations to be located. 
J. Calle, et al, The standard refuelling facility expansion problem: A case study in Australia

Locate new stations: this scenario adds the potential locations of the new stations into the model.

Locate maximum one station: this scenario adds the constraint that only one station can be built to the Locate new stations scenario.

$\sum_{i \in \mathcal{S}^{P O T}} y_{i}=1$

\subsection{Results}

Table 2 presents the total cost of each scenario and the percentage of savings with respect to the baseline. It is observed that just by improving the refuelling decisions, without investing in new stations, the company could saved $2.3 \%$. It means that the location of two additional facilities, one in Tarcutta and one in Marulan, would add $2.5 \%$ of additional savings.

Table 2. Total cost and location decisions per scenario

\begin{tabular}{|c|c|c|c|}
\hline Scenario & Total cost $(\mathbf{z})$ & Savings & Location decisions \\
\hline Baseline & $\$ 30,821,247$ & $0.0 \%$ & - \\
\hline Optimised baseline & $\$ 30,104,462$ & $2.3 \%$ & - \\
\hline Locate new stations & $\$ 29,354,070$ & $4.8 \%$ & Tarcutta + Marulan \\
\hline Locate max one station & $\$ 29,525,393$ & $4.2 \%$ & Tarcutta \\
\hline
\end{tabular}

To test the resilience of the location of two stations in the corridor, we ran a sensitivity analysis, in which the total fuel consumed in the corridor was reduced at different levels. This is done by multiplying the parameter $Q_{v, p}^{v e h}$ by the corresponding reducing factor $(0.9,0.8,0.7,0.6,0.5,0.4$ and 0.3$)$ of each scenario. Table 3 presents the results of this exercise.

It can be seen that a reduction of $20 \%$ of fuel consumed in the corridor will generate that the optimal solution would be just to locate one station in Tarcutta. It is observed that locating only one station in Tarcutta is still the optimal solution even with a reduction of $60 \%$ of fuel consumed in the corridor. A reduction of $70 \%$ of fuel consumed in the corridor will make that locating a station would not be profitable.

If the company decided to locate only one station at Tarcutta, the model suggests to refuel there around 11.7 million litres per year. This means that it is necessary to increase the standard capacity of 9.4 million litres to 11.7 million litres per year. Recall that the capacity is increased in multiples of 2.3 million litres per year.

Table 3. Sensitivity analysis. Fuel consumption reduction in the corridor Melbourne-Sydney

\begin{tabular}{|l|c|c|c|c|c|c|c|c|}
\cline { 3 - 9 } \multicolumn{2}{c|}{} & \multicolumn{9}{|c|}{ Scenario } \\
\cline { 3 - 9 } & Baseline & \multicolumn{2}{c|}{ Locate new stations } & \multicolumn{3}{c|}{ Locate max 1 station } \\
\hline $\begin{array}{c}\text { \% } \\
\text { Reduction }\end{array}$ & $\begin{array}{c}\text { Consumption } \\
\text { (litres) }\end{array}$ & Total cost & Total cost & Savings & $\begin{array}{c}\text { Location } \\
\text { decision }\end{array}$ & Total cost & Savings & $\begin{array}{c}\text { Location } \\
\text { decision }\end{array}$ \\
\hline $0 \%$ & $21,107,259$ & $\$ 30,821,247$ & $\$ 29,354,070$ & $4.8 \%$ & $\begin{array}{c}\text { Tarcutta }+ \\
\text { Marulan }\end{array}$ & $\$ 29,525,393$ & $4.2 \%$ & Tarcutta \\
\hline$-10 \%$ & $18,996,533$ & $\$ 27,739,558$ & $\$ 26,439,556$ & $4.7 \%$ & $\begin{array}{l}\text { Tarcutta }+ \\
\text { Marulan }\end{array}$ & $\$ 26,586,024$ & $4.2 \%$ & Tarcutta \\
\hline$-20 \%$ & $16,885,807$ & $\$ 24,657,246$ & $\$ 23,624,673$ & $4.2 \%$ & Tarcutta & $\$ 23,624,673$ & $4.2 \%$ & Tarcutta \\
\hline$-30 \%$ & $14,775,081$ & $\$ 21,575,062$ & $\$ 20,684,545$ & $4.1 \%$ & Tarcutta & $\$ 20,684,545$ & $4.1 \%$ & Tarcutta \\
\hline$-40 \%$ & $12,664,355$ & $\$ 18,493,080$ & $\$ 17,745,176$ & $4.0 \%$ & Tarcutta & $\$ 17,745,176$ & $4.0 \%$ & Tarcutta \\
\hline$-50 \%$ & $10,553,629$ & $\$ 15,410,945$ & $\$ 14,805,806$ & $3.9 \%$ & Tarcutta & $\$ 14,805,806$ & $3.9 \%$ & Tarcutta \\
\hline$-60 \%$ & $8,442,903$ & $\$ 12,328,797$ & $\$ 11,866,437$ & $3.8 \%$ & Tarcutta & $\$ 11,866,437$ & $3.8 \%$ & Tarcutta \\
\hline$-70 \%$ & $6,332,178$ & $\$ 9,246,565$ & $\$ 9,031,339$ & $2.3 \%$ & - & $\$ 9,031,339$ & $2.3 \%$ & - \\
\hline
\end{tabular}

\section{Conclusions}

Given the data available to make this study, the model suggests that, by improving the refuelling decision taken by the fleet, savings of $2.3 \%$ could be obtained, which in the studied corridor would represent around $\$ 700,000$ per year $^{2}$. It is important to notice that the model suggests an optimal refuelling pattern for each 
J. Calle, et al, The standard refuelling facility expansion problem: A case study in Australia

type of vehicle based solely on financial and logistic factors. Other factors, such as the personal preferences of drivers - who at the end are the ones that make the refuelling decisions - are beyond the scope of the model.

A robust site to locate a station is Tarcutta, which will yield savings with respect to the baseline of $4.2 \%$ and around $1.9 \%$ with respect to the optimised baseline. Opening a station at this site remains a financially sound decision even with a reduction of $60 \%$ in the fuel consumed in the corridor, as shown by the sensitivity analysis presented in Table 3. This solution is more robust than locating two stations, as a reduction of $20 \%$ of the volume in the corridor would make that the best is to locate just one station.

From a theoretical perspective, the MIP model presented in this paper is new in the flow refuelling location literature, in that it takes into account the differential price of the fuel in each station as a factor in the location decision. Another contribution is the novel procedure presented in this paper to process and convert large amounts of GPS data into trips. Such an approach was until now absent in the literature.

\section{Future RESEARCH}

One of the main parameters that affects the fuel consumed in the corridor was the quantity of vehicles travelling through each path $Q_{v, p}^{v e h}$. To address the natural uncertainty of this parameter, a sensitivity analysis is run over the fuel consumed in the corridor. However, for future research it would be interesting to approach this problem having into account the quantity of vehicles travelling through each path as a stochastic parameter instead of a deterministic parameter.

\section{ACKNOWLEDGEMENTS}

The authors acknowledge the support of the anonymous transport company which provided the data and cofunded with Deakin University the Ph.D. studies of the presenting author. The authors thank Patricia Mclean and the reviewers for their valuable comments in the final manuscript.

\section{REFERENCES}

Calle, J., Perez-Franco, R., Mak-Hau, V., Abril, D., \& Rojas, D. (2021). Supplementary material. https://bit. ly/3sGZY2R

Deb, S., Tammi, K., Kalita, K., \& Mahanta, P. (2018). Review of recent trends in charging infrastructure planning for electric vehicles. Wiley Interdisciplinary Reviews: Energy and Environment, 7(6).

https: //doi.org/10.1002/wene.306

Kuby, M., \& Lim, S. (2005). The flow-refueling location problem for alternative-fuel vehicles. Socio-Economic Planning Sciences, 39(2), 125-145. https://doi.org/10.1016/j.seps.2004.03.001

MirHassani, S. A., \& Ebrazi, R. (2013). A Flexible Reformulation of the Refueling Station Location Problem. Transportation Science, 47(4), 617-628. https://doi.org/10.1287/trsc.1120.0430

Suzuki, Y. (2008). A generic model of motor-carrier fuel optimization. Naval Research Logistics, 55(8), 737746. https://doi.org/10.1002/nav.20317

Thakur, A., Pinjari, A. R., Zanjani, A. B., Short, J., Mysore, V., \& Tabatabaee, S. F. (2015). Development of algorithms to convert large streams of truck GPS data into truck trips. Transportation Research

Record, 2529, 66-73. https://doi.org/10.3141/2529-07

Wang, Y.-W., \& Lin, C.-C. (2009). Locating road-vehicle refueling stations. Transportation Research Part E: Logistics and Transportation Review, 45(5), 821-829. https://doi.org/10.1016/j.tre.2009.03.002

Watson, M., Lewis, S., Cacioppi, P., \& Jayaraman, J. (2013). Supply Chain Network Design: Applying Optimization and Analytics to the Global Supply Chain. FT Press.

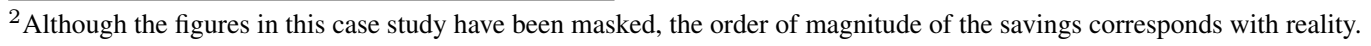

